\title{
Josephson radiation in a superconductor-quantum dot-superconductor junction
}

\author{
Baptiste Lamic $\odot$, Julia S. Meyer, and Manuel Houzet $\odot$ \\ Univ. Grenoble Alpes, CEA, IRIG-Pheliqs, F-38000 Grenoble, France
}

(Received 4 March 2020; revised 26 June 2020; accepted 29 June 2020; published 28 July 2020)

\begin{abstract}
We investigate the Josephson radiation emitted by a junction made of a quantum dot coupled to two conventional superconductors. At strong coupling, such a junction accommodates two Andreev bound states, which are slightly detached from the continuum outside the superconducting gap. Furthermore, when the dot is tuned close to resonance, the residual backscattering opens a small gap near the Fermi energy between the discrete states. Under voltage bias, we formulate a stochastic model that accounts for nonadiabatic processes, which change the occupations of the Andreev bound states. This model allows one to calculate the current noise spectrum and determine the Fano factor. Analyzing the finite-frequency noise, we find that the model may exhibit either an integer or a fractional AC Josephson effect, depending on the bias voltage and the size of the gaps in the Andreev spectrum. Our results assess the limitations in using the fractional Josephson radiation as a probe of topological superconductivity.
\end{abstract}

DOI: 10.1103/PhysRevResearch.2.033158

The Josephson radiation is the electromagnetic signal emitted by a junction between two superconductors when it is voltage biased. Its measurement is the first experimental demonstration of the AC Josephson effect [1]. The charge of the Cooper pairs, which form the superconducting condensate, appears in the relation between the radiation frequency and voltage bias, $\omega=\omega_{J}$, where $\omega_{J}=2 \mathrm{eV} / \hbar$ is the Josephson frequency. The coherence property of the Josephson radiation is usually limited by the electromagnetic environment of the junction. Tailoring the environment surrounding a superconducting tunnel junction recently allowed the regime of Josephson lasing to be reached [2].

Interest in the Josephson radiation was revived by the prediction of a fractional AC Josephson effect at frequency $\omega=$ $\omega_{J} / 2$ when the superconducting leads forming the junction are topological superconductors [3-6]. This fractional Josephson radiation originates from the fact that the supercurrent flowing through such a topological Josephson junction is carried by single electrons, rather than Cooper pairs. The single-electron transfer is made possible by the hybridization of Majorana end modes in the topological superconducting leads. A topological junction admits two degenerate parity sectors, carrying Josephson supercurrents with opposite values. Therefore, random parity switchings generate current noise, and eventually bring another limitation to the coherence of the fractional Josephson radiation [7].

On a microscopic level, the Josephson effect can be associated with the formation of subgap states, also known as Andreev bound states (ABS), in the junction. The difference between conventional and topological Josephson junctions is

Published by the American Physical Society under the terms of the Creative Commons Attribution 4.0 International license. Further distribution of this work must maintain attribution to the author(s) and the published article's title, journal citation, and DOI. associated with the fact that in equilibrium, the ABS energy depends $2 \pi$ periodically on the superconducting phase difference $\varphi$ in the conventional case, while the dependence is $4 \pi$ periodic in the topological case. Then the integer or fractional Josephson radiations simply result from the substitution $\dot{\varphi}=$ $\omega_{J}$ in the phase dependence of the current carried by an ABS whose occupation is fixed. However, such considerations neglect the voltage-induced nonadiabatic processes that change the ABS occupations and, therefore, reduce the coherence of the Josephson radiation, even when the effect of the external environment is negligible. Indeed, it was shown that such nonadiabatic processes in topological junctions introduce an intrinsic limitation to the visibility of the fractional Josephson radiation [8-10]. This mechanism is ultimately related with the dissipative current that flows through the junction, which is induced by these processes.

In this work, we investigate the role of nonadiabatic processes on the Josephson radiation in a nontopological junction formed by a quantum dot connected to conventional superconducting leads. We find that such conventional junctions may display either a conventional or a fractional Josephson radiation as the voltage varies, depending on details of the ABS spectrum. In particular, a fractional Josephson radiation is predicted when the gap in the Andreev spectrum near the Fermi level is crossed diabatically $[11,12]$, while the ABS are sufficiently detached from the continuum above the superconducting gap. Furthermore, we determine the contribution of the nonadiabatic transitions to the linewidth of the Josephson radiation. Our results assess the limitations in using the current noise spectrum to determine whether or not a Josephson junction is topological.

We consider a junction made of a spin-degenerate singlelevel quantum dot that is contacted to two superconducting leads. We first recall the properties of its Andreev spectrum, which have been analyzed both in the presence and absence of Coulomb interaction; see Refs. [13-15]. Let us first start with the case of strong Coulomb interaction. It allows for 


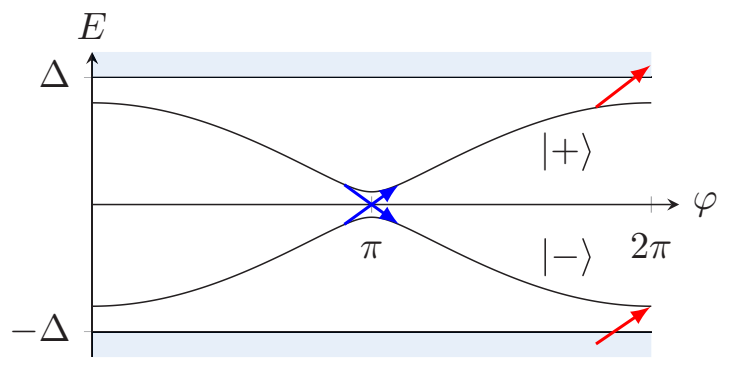

FIG. 1. Spectrum of the Andreev bound states as a function of the superconducting phase difference. In the presence of a voltage bias, the phase increases with time and nonadiabatic transitions may occur between states $|+\rangle$ and $|-\rangle$ (red arrows), as well as between state $|+\rangle$ and the continuum at energy $E>\Delta$, or between the continuum at energy $E<-\Delta$ and state $|-\rangle$ (red arrows).

resonant scattering of electrons between the leads in the normal state thanks to the Kondo effect. For energies smaller than the Kondo scale $T_{K}$, the transmission probability is $T_{\pi}=$ $4 \Gamma_{L} \Gamma_{R} / \Gamma^{2}$, where $\Gamma=\Gamma_{L}+\Gamma_{R}$, with $\Gamma_{L}$ and $\Gamma_{R}$ the partial level widths due to the coupling of the dot to the left and right leads. For an almost symmetric coupling, the transmission is almost ballistic, $R_{\pi} \equiv 1-T_{\pi} \ll 1$. When the leads are superconducting and the superconducting gap is sufficiently small, $\Delta \ll T_{K}$, the junction accommodates two particle-hole symmetric ABS denoted $|+\rangle$ and $|-\rangle$. Their energies are approximated by

$$
E_{ \pm}(\varphi)= \pm \Delta \sqrt{T_{0} \cos ^{2}(\varphi / 2)+R_{\pi} \sin ^{2}(\varphi / 2)} .
$$

In addition to the gap opening in the Andreev spectrum near $\varphi=\pi$ at $T_{\pi}<1$ [16], Eq. (1) also shows the detachment of the ABS from the edge of the continuum spectrum near $\varphi=0[17,18]$, which is controlled by an effective reflection probability $R_{0} \equiv 1-T_{0}$ of the order of $\left(\Delta / T_{K}\right)^{2} \ll 1$ (up to a logarithmic correction [19]). In Ref. [18], the gap opening near $\varphi=0$ was related with residual local interaction in the Fermi-liquid description of the Kondo effect. Interestingly, Eq. (1) also holds in the absence of interactions, after substituting $T_{K}$ with $\Gamma \gg \Delta$ [20] in the estimate for $R_{0}$. The ABS dispersion is illustrated in Fig. 1.

We turn now to the current flowing through the junction. Each occupied ABS carries a supercurrent,

$$
I_{ \pm}(\varphi)=\frac{2 e}{\hbar} \frac{\partial E_{ \pm}(\varphi)}{\partial \varphi} \approx \mp I_{J} \sin \frac{\varphi}{2} \operatorname{sgn}\left(\cos \frac{\varphi}{2}\right),
$$

where $I_{J}=e \Delta / \hbar$, and we used $R_{0}, R_{\pi} \ll 1$ in the last equality. In equilibrium, the average ABS occupations are set by the Fermi distribution, while the contribution of the continuum is negligibly small. Thus the equilibrium supercurrent at zero temperature is given by $I_{-}(\varphi)$.

In the presence of a DC voltage bias, the phase difference increases linearly with time, $\varphi(t)=\omega_{J} t+\varphi_{0}$, with a reference phase $\varphi_{0}$. (Here we assume $V>0$, for concreteness.) As a consequence, changes in the occupations of the ABS can occur due to nonadiabatic transitions. Using $R_{0}, R_{\pi} \ll 1$ and $V \ll \Delta / e$, we can isolate two kinds of nonadiabatic processes. Near $\varphi=\pi \bmod 2 \pi$, these are the transitions between $|+\rangle$ and $|-\rangle$, which occur with the Landau-Zener probability $p_{\pi}=\exp \left(-\pi R_{\pi} \Delta / e V\right)[21] ; p_{\pi}$ increases rapidly from 0 to 1 as $V$ increases in the vicinity of $V_{\pi}=R_{\pi} \Delta / e$. Near $\varphi=0 \bmod 2 \pi$, these are nonadiabatic transitions between $|+\rangle$ and the continuum states with energy $E>\Delta$ as well as the continuum states with energy $E<-\Delta$ and $|-\rangle$ [22], which take place with probability $p_{0}=p\left(V / V_{0}\right)$ with $V_{0}=R_{0}^{3 / 2} \Delta / e$, where the function $p(x)$ calculated in Ref. [23] (and Ref. [22] at $\left.V \ll V_{0}\right)$ is such that $p_{0}$ increases rapidly from 0 to 1 as $V$ increases in the vicinity of $V_{0}$.

We assume $T \ll \Delta$, so that continuum states with energy $E<-\Delta(E>\Delta)$ are occupied (empty). We also neglect the short timescales over which the nonadiabatic processes take place on the scale of the Josephson period, $2 \pi / \omega_{J}$. Then, at each time, the state of the junction is fully characterized by the occupations $n_{ \pm}=0,1$. (In particular, we ignore coherent superpositions between $| \pm\rangle$ states.) The states $(0,1)$ and $(1,0)$ are the ground and first-excited states in the even-parity sector of the junction, respectively; the states $(0,0)$ and $(1,1)$ are the "poisoned" states in the odd-parity sector [24,25]. Within a Markov model that describes switchings in their random occupations $[23,26]$, the average supercurrent is

$$
\langle I(t)\rangle=\mathcal{I}(t)\left[P_{01}(\varphi(t))-P_{10}(\varphi(t))\right] .
$$

Here, $\mathcal{I}(t)=I_{-}(\varphi(t))$ and $P_{n_{+} n_{-}}(\varphi)$ with $n_{+}, n_{-}=0,1 \mathrm{de}-$ notes the ABS occupations at a given phase. Neglecting any coupling with an external bath, these probabilities remain constant within intervals $\pi m<\varphi<\pi(m+1)$ with $m$ integer, while their values immediately before and after the specific phases where nonadiabatic transitions can take place are related with each other through the transition probabilities $p_{\pi}$ and $p_{0}$,

$$
\begin{aligned}
\boldsymbol{P}\left[(2 m+1) \pi^{+}\right] & =\mathcal{L}_{\pi} \boldsymbol{P}\left[(2 m+1) \pi^{-}\right], \\
\boldsymbol{P}\left(2 m \pi^{+}\right) & =\mathcal{L}_{0} \boldsymbol{P}\left(2 m \pi^{-}\right),
\end{aligned}
$$

with $\boldsymbol{P}(\varphi)=\left[P_{11}(\varphi), P_{10}(\varphi), P_{01}(\varphi), P_{00}(\varphi)\right]^{T}$ and the transition matrices

$$
\begin{aligned}
\mathcal{L}_{\pi} & =\left(\begin{array}{cccc}
1 & 0 & 0 & 0 \\
0 & 1-p_{\pi} & p_{\pi} & 0 \\
0 & p_{\pi} & 1-p_{\pi} & 0 \\
0 & 0 & 0 & 1
\end{array}\right), \\
\mathcal{L}_{0} & =\left(\begin{array}{cccc}
1-p_{0} & p_{0}\left(1-p_{0}\right) & 0 & 0 \\
0 & \left(1-p_{0}\right)^{2} & 0 & 0 \\
p_{0} & p_{0}^{2} & 1 & p_{0} \\
0 & p_{0}\left(1-p_{0}\right) & 0 & 1-p_{0}
\end{array}\right) .
\end{aligned}
$$

The nonadiabatic processes accounted for by the matrix elements of $\mathcal{L}_{\pi}$ and $\mathcal{L}_{0}$ are illustrated in Figs. 2(b) and 2(c).

The stochastic matrix $\mathcal{L} \equiv \mathcal{L}_{0} \mathcal{L}_{\pi}$ admits normalized right and left eigenvectors $u_{\alpha}$ and $v_{\alpha}$, with a common eigenvalue $\lambda_{\alpha}$, such that $\mathcal{L} u_{\alpha}=\lambda_{\alpha} u_{\alpha}, \mathcal{L}^{T} v_{\alpha}=\lambda_{\alpha} v_{\alpha}$, and $v_{\alpha}^{T} u_{\beta}=\delta_{\alpha \beta}$. Furthermore, the eigenvalue $\lambda_{0}=1$ is associated with the left eigenvector $v_{0}=(1, \ldots, 1)^{T}$, while other eigenvalues, $\lambda_{1}=$ $1-p_{0}, \lambda_{2}=\left(1-p_{0}\right)^{2}$, and $\lambda_{3}=\left(1-p_{0}\right)\left(1-2 p_{\pi}\right)$, satisfy $\left|\lambda_{\alpha \neq 0}\right|<1$. Thus, the probability vector $\boldsymbol{P}$ that solves Eqs. (1) reaches a solution at long times that does not depend on the initial condition; it is given by the eigensolution $\alpha=0$ :

$$
\boldsymbol{P}(\varphi)= \begin{cases}u_{0}, & 2 m \pi<\varphi<(2 m+1) \pi, \\ \mathcal{L}_{\pi} u_{0}, & (2 m+1) \pi<\varphi<(2 m+2) \pi,\end{cases}
$$


(a)
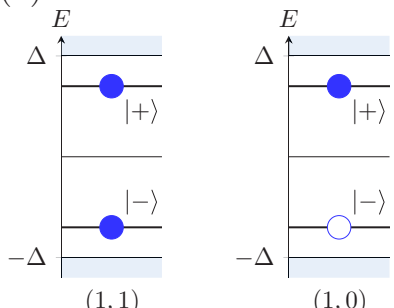

$(1,0)$

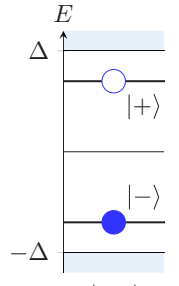

$(0,1)$

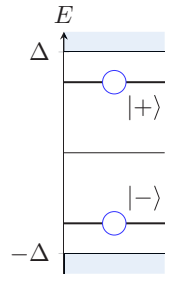

$(0,0)$

(b)

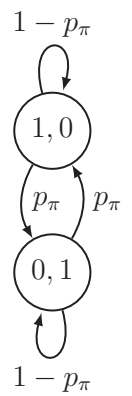

(c)

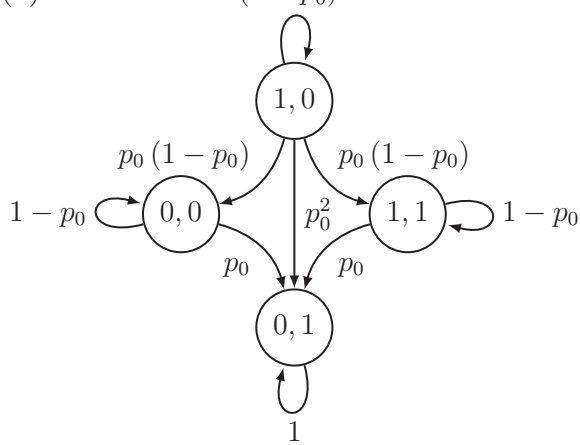

FIG. 2. (a) The junction state is characterized by the occupations of the two Andreev bound states. Filled and unfilled circles depict occupied and unoccupied Andreev bound states, respectively. (b),(c) Graphs of the transition matrices. Each arrow denotes a possible transition between two states $\left(n_{+}, n_{-}\right)$and $\left(n_{+}^{\prime}, n_{-}^{\prime}\right)$, and is labeled by the transition probability. (b) Nonadiabatic processes described by $\mathcal{L}_{\pi}$ can exchange the occupation of states $(0,1)$ and $(1,0)$ by enabling a particle from one ABS to jump to the other ABS. (c) Nonadiabatic processes described by $\mathcal{L}_{0}$ can populate the lower ABS, enabling transitions from $(0,0)$ to $(0,1)$, and from $(1,0)$ to $(1,1)$. They can also deplete the higher ABS state, enabling transitions from $(1,0)$ to $(0,0)$ and from $(1,1)$ to $(0,1)$. As the changes of the population of the lower and upper ABS are independent, the total transition probabilities indicated in the figure are a product of two probabilities.

with

$$
\begin{aligned}
u_{0} & =\frac{1}{\mathcal{N}}\left(\begin{array}{c}
p_{\pi}\left(1-p_{0}\right) \\
p_{\pi}\left(1-p_{0}\right)^{2} \\
\mathcal{N}-p_{\pi}\left(1-p_{0}\right)\left(3-p_{0}\right) \\
p_{\pi}\left(1-p_{0}\right)
\end{array}\right), \\
\mathcal{L}_{\pi} u_{0} & =\frac{1}{\mathcal{N}}\left(\begin{array}{c}
p_{\pi}\left(1-p_{0}\right) \\
p_{\pi} \\
\mathcal{N}-p_{\pi}\left(3-2 p_{0}\right) \\
p_{\pi}\left(1-p_{0}\right)
\end{array}\right),
\end{aligned}
$$

and $\mathcal{N}=\left(2-p_{0}\right)\left(1-\lambda_{3}\right)$. In particular, the ground state $(0,1)$ is mostly occupied with $u_{0} \approx \mathcal{L}_{\pi} u_{0} \approx(0,0,1,0)^{T}$ at $p_{\pi} \ll p_{0}$, while all states are approximately equally occupied with $u_{0} \approx \mathcal{L}_{\pi} u_{0} \approx\left(\frac{1}{4}, \frac{1}{4}, \frac{1}{4}, \frac{1}{4}\right)^{T}$ at $p_{0} \ll p_{\pi}$.

Inserting Eq. (6) into (3), we find the average current in the long-time limit,

$$
\begin{aligned}
\langle I(t)\rangle= & \frac{p_{0}}{1-\lambda_{3}}\left[p_{\pi}|\mathcal{I}(t)|+\left(1-p_{\pi}\right) \mathcal{I}(t)\right] \\
= & I_{\mathrm{DC}}\left(1-\sum_{n \geqslant 1} \frac{2}{4 n^{2}-1}\{\cos [n \varphi(t)]\right. \\
& \left.\left.+2(-1)^{n} n \frac{1-p_{\pi}}{p_{\pi}} \sin [n \varphi(t)]\right\}\right) .
\end{aligned}
$$

The DC contribution,

$$
I_{\mathrm{DC}}=\frac{2}{\pi} \frac{p_{0} p_{\pi}}{1-\lambda_{3}} I_{J}
$$

relates the dissipative current with nonadiabatic processes through the gaps in the Andreev spectrum. It corresponds to the low-voltage regime of multiple Andreev reflections (MAR). It generalizes formulas derived in superconducting atomic contacts [21] at $p_{0}=1$, and in topological Josephson junctions [23] at $p_{\pi}=1$. The ratio between cosine and sine harmonics of the Josephson frequency is controlled by nonadiabatic transitions between ABS with positive and negative energies. When these processes are rare, the sine harmonics dominate, such as in the adiabatic case.

Due to the stochastic nature of the nonadiabatic processes, the current fluctuates. We characterize the fluctuations with the current noise spectrum,

$$
S(\omega)=2 \int_{0}^{\infty} d \tau \cos (\omega \tau) \overline{S(t+\tau / 2, t-\tau / 2)},
$$

where the bar denotes an average over $t$. Within the Markov theory, we relate the current correlator,

$$
S\left(t_{1}, t_{2}\right)=\left\langle I\left(t_{1}\right) I\left(t_{2}\right)\right\rangle-\left\langle I\left(t_{1}\right)\right\rangle\left\langle I\left(t_{2}\right)\right\rangle,
$$

with

$$
\begin{aligned}
& \left\langle I\left(t_{1}\right) I\left(t_{2}\right)\right\rangle \\
& \quad=\mathcal{I}\left(t_{1}\right) \mathcal{I}\left(t_{2}\right) \sum_{n_{1}, n_{2}}(-1)^{n_{1}+n_{2}} P_{n_{1} \bar{n}_{1} \mid n_{2} \bar{n}_{2}}\left(\varphi_{1} \mid \varphi_{2}\right) P_{n_{2} \bar{n}_{2}}^{\infty}\left(\varphi_{2}\right)
\end{aligned}
$$

at $t_{1}>t_{2}$. Here, $P_{n_{1} n_{1}^{\prime} \mid n_{2} n_{2}^{\prime}}\left(\varphi_{1} \mid \varphi_{2}\right)$ with $\varphi_{i}=\varphi\left(t_{i}\right)$ is the conditional probability for the system to reside in state $\left(n_{1}, n_{1}^{\prime}\right)$ at phase $\varphi_{1}$ if it was in state $\left(n_{2}, n_{2}^{\prime}\right)$ at phase $\varphi_{2}<\varphi_{1}$; it solves the same Eq. (4) as the probability $P_{n_{1} n_{1}^{\prime}}\left(\varphi_{1}\right)$, together with the initial condition $P_{n_{1} n_{1}^{\prime} \mid n_{2} n_{2}^{\prime}}\left(\varphi_{2} \mid \varphi_{2}\right)=\delta_{n_{1}, n_{2}} \delta_{n_{1}^{\prime}, n_{2}^{\prime}}$. Furthermore, we used notations $\overline{0}=1$ and $\overline{1}=0$. Using a matrix representation [in the same basis of states as the one used in Eqs. (4) and (5)] for the closure relation, $\sum_{\alpha} u_{\alpha} v_{\alpha}^{T}=1$, we find

$$
P\left(\varphi_{1} \mid \varphi_{2}\right)=\mathcal{L}_{\pi}^{k_{1}}\left(\sum_{\alpha} u_{\alpha} \lambda_{\alpha}^{m_{1}-m_{2}} v_{\alpha}^{T}\right) \mathcal{L}_{\pi}^{-k_{2}},
$$

for $\left(2 m_{i}+k_{i}\right) \pi<\varphi_{i}<\left(2 m_{i}+k_{i}+1\right) \pi$, with $m_{i}$ integer, $k_{i}=0,1$, and $i=1,2$. The second term in the right-hand side of Eq. (11) compensates the contribution from the terms with $\alpha=0$ when inserting Eq. (13) into (12). Furthermore, the expectation value of the current operator in the states with $\alpha=$ 1,2 vanishes. Therefore, only the terms with $\alpha=3$ contribute to Eq. (11). As the result of a long calculation described in Sec. S1 of the Supplemental Material (SM) [27], we find

$$
\frac{S(\omega)}{S_{0}}=\frac{A\left[1+4 \tilde{\omega}^{2}-4 \tilde{\omega} \sin (\pi \tilde{\omega})\right]+\left(B+C \tilde{\omega}^{2}\right) \cos ^{2}(\pi \tilde{\omega})}{\left(1-4 \tilde{\omega}^{2}\right)^{2}\left[\left(1+\lambda_{3}\right)^{2}-4 \lambda_{3} \cos ^{2}(\pi \tilde{\omega})\right]},
$$

with $S_{0}=I_{J}^{2} /\left(\pi \omega_{J}\right), \tilde{\omega}=\omega / \omega_{J}$, and

$$
\begin{aligned}
& A=8 p_{0} p_{\pi}\left(1-p_{\pi}\right)\left[1+\left(1-p_{0}\right)^{2}\right]\left(1+\lambda_{3}\right) / \mathcal{N}, \\
& B=-16 p_{\pi}\left(2-p_{\pi}\right) \lambda_{3} / \mathcal{N}, \\
& C=128 p_{\pi}\left(1-p_{\pi}\right)\left(1-p_{0}\right)\left[1-\lambda_{3}\left(1-p_{0}\right)\right] / \mathcal{N} .
\end{aligned}
$$




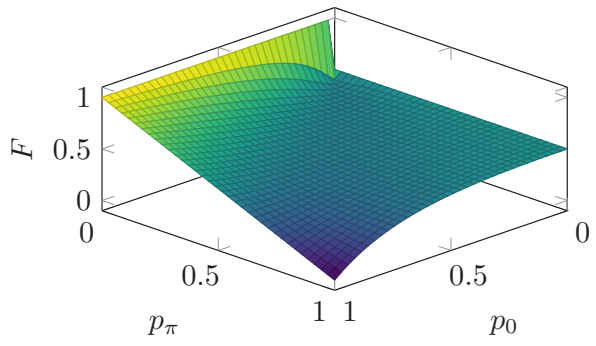

FIG. 3. Fano factor as a function of $p_{0}$ and $p_{\pi}$.

Below we discuss the zero-frequency noise as well as structures related to the AC currents in the frequency dependence of the noise given by Eq. (14).

The zero-frequency noise is expressed in terms of an effective charge, $e^{\star}=2 \Delta / V$, which diverges inversely with the bias voltage in the MAR regime, and a Fano factor

$$
F \equiv \frac{S(0)}{e^{\star} I_{\mathrm{DC}}}=\frac{A+B}{8 p_{0} p_{\pi}\left(1-\lambda_{3}\right)} .
$$

In particular, $F=1$ at $p_{\pi} \ll p_{0}$, when the bottleneck for the transfer of quasiparticles across the junction is the gap near the Fermi level [28]; $F=1 / 2$ at $p_{0} \ll p_{\pi}$, when the bottleneck consists of the two gaps (of the same width) near the edges of the continuum spectrum. In particular, at $p_{\pi}=1$, on recovers the results of Ref. [23] for a topological junction (see Sec. $\mathrm{S} 2$ of the $\mathrm{SM}[27]) . F$ vanishes at $1-p_{0}, 1-p_{\pi} \ll 1$, when quasiparticle transfer across the gap becomes deterministic. In the general case, $0<F<1$; see Fig. 3 .

From Eq. (14), we see that the noise displays sharp features when $1 \mp \lambda_{3} \ll 1$. This is the case when $p_{0}, p_{\pi} \ll 1$ or $p_{0}, 1-p_{\pi} \ll 1$. We turn now to the noise spectrum in these limiting cases for the transition probabilities.

At $p_{0}, p_{\pi} \ll 1$, the noise spectrum displays features with a narrow linewidth,

$$
\gamma=\frac{1}{2 \pi}\left(p_{0}+2 p_{\pi}\right) \omega_{J}
$$

near $\omega=n \omega_{J}$ with $n$ integer. In particular, at very low frequency, $\omega \ll \omega_{J}$,

$$
\frac{S(\omega)}{e^{\star} I_{\mathrm{DC}}}=F+\left(F_{\mathrm{app}}-F\right) \frac{\omega^{2}}{\omega^{2}+\gamma^{2}} .
$$

Here, Eq. (9) simplifies to

$$
I_{\mathrm{DC}}=\frac{2 I_{J}}{\pi} \frac{p_{0} p_{\pi}}{p_{0}+2 p_{\pi}},
$$

and the Fano factor,

$$
F=\frac{p_{0}^{2}+p_{0} p_{\pi}+2 p_{\pi}^{2}}{\left(p_{0}+2 p_{\pi}\right)^{2}},
$$

is only accessible in a narrow frequency range, $\omega \ll \gamma$, while an apparent Fano factor,

$$
F_{\text {app }}=\frac{\left(12-4 \pi+\pi^{2}\right) p_{0}+8 p_{\pi}}{2 \pi^{2} p_{0}},
$$

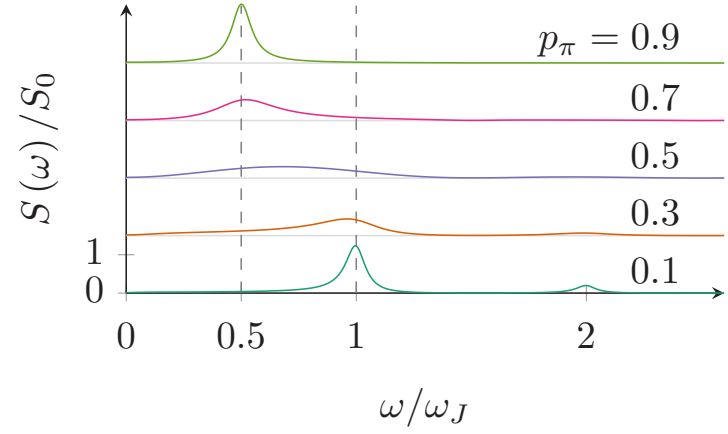

FIG. 4. Current noise spectrum for $p_{0}=0.1$ and several equally spaced values of $p_{\pi}$ between 0.1 and 0.9 . The curves are shifted vertically for clarity.

characterizes the noise in a wide frequency range, $\gamma \ll \omega \ll$ $\omega_{J}$. Note that $F_{\text {app }} \gg F$ if $p_{0} \ll p_{\pi}$. Furthermore,

$$
S(\omega)=\frac{32 n^{2}}{\left(4 n^{2}-1\right)^{2} \pi} \frac{\left(3 p_{0}+2 p_{\pi}\right) p_{\pi}}{\left(p_{0}+2 p_{\pi}\right)^{2}} \frac{\gamma I_{J}^{2} / \pi}{\left(\omega-n \omega_{J}\right)^{2}+\gamma^{2}}
$$

at $\left|\omega-n \omega_{J}\right| \ll \omega_{J}$, up to a negative resonance-frequency shift of the order of $\gamma^{2} / \omega_{J} \ll \gamma$. Comparing Eq. (22) with the amplitude of the harmonics in Eq. (8), we conclude that the Josephson radiation at $p_{0} \ll p_{\pi} \ll 1$ is dominated by the noise, given by Eq. (22); thus it is broadened by nonadiabatic transitions. On the other hand, the Josephson radiation at $p_{\pi} \ll p_{0} \ll 1$ is dominated by the sine harmonics in Eq. (8); thus it is broadened by the environment of the junction.

At $p_{0}, 1-p_{\pi} \ll 1, I_{\mathrm{DC}}=p_{0} I_{J} / \pi$ and $F=1 / 2$; the noise spectrum displays a narrow resonance at half the Josephson frequency,

$$
S(\omega)=\frac{I_{J}^{2}}{4} \frac{\gamma^{\prime}}{\left(\omega-\omega_{J} / 2\right)^{2}+\gamma^{\prime 2}},
$$

with linewidth

$$
\gamma^{\prime}=\frac{1}{2 \pi}\left[p_{0}+2\left(1-p_{\pi}\right)\right] \omega_{J},
$$

up to a small resonance-frequency shift, of the order of $\gamma^{\prime 2} / \omega_{J} \ll \gamma^{\prime}$, which increases as $p_{\pi}$ decreases. Higher-order resonances around $(n+1 / 2) \omega_{J}$ are suppressed. Comparison between Eqs. (8) and (24) shows that the Josephson radiation is dominated by the noise, and it is thus broadened by the nonadiabatic processes. At $1-p_{\pi} \ll 1$, transitions across the gap at $\pi$ are very frequent, leading to a large, but random $4 \pi$-periodic contribution to the current. Thus, the Josephson radiation is fractional despite the junction not being topological. In the extreme case $p_{\pi}=1$, where the transitions across the gap at $\pi$ are deterministic, the system can be described as two independent topological junctions in parallel $[18,27]$.

The crossover between a well-resolved fractional or conventional Josephson radiation, when $p_{0}$ is small and $p_{\pi}$ increases from 0 to 1 , occurs through the gradual shift and broadening of the peaks in the noise spectrum, as illustrated in Fig. 4. A similar behavior has been reported in topological junctions, but with a residual coupling to the Majoranas at the far ends of the superconducting wires [9]. 
In a given junction, we can now distinguish two qualitatively different behaviors, depending on the ratio between crossover voltages $V_{0}$ and $V_{\pi}$. If $V_{0} \ll V_{\pi}, F=1$ at $V \ll V_{\pi}$, then it drops to 0 at $V \gg V_{\pi}$. Furthermore, the Josephson radiation is conventional. In the opposite case, $V_{\pi} \ll V_{0}, F=1 / 2$ at $V \ll V_{0}$; then it drops to 0 at $V \gg V_{0}$, and there is a voltage range $V_{\pi} \ll V \ll V_{0}$ where Josephson radiation is fractional. Both the conventional and fractional Josephson radiations are broadened by the nonadiabatic processes at $V<V_{0}$.

In conclusion, we proposed a simple model to analyze the role of nonadiabatic transitions between Andreev states in the Josephson radiation of a superconductor-quantum dotsuperconductor junction. Within a simplified model of the Andreev states' dynamics, we predicted that such a conventional junction may display either conventional radiation or fractional radiation, thus mimicking a topological Josephson junction, depending on its parameters and on the bias voltage. On the theory side, it would be interesting to extend the analysis to a more general description of the Andreev dynamics, as well as to develop a theory frame to compare the interplay of the environment (neglected in our study) and the nonadiabatic transition on the loss of coherence of the Josephson radiation. On the experimental side, it would be interesting to compare our prediction with finite-frequency noise measurements in devices such as the superconductorcarbon nanotube-superconductor junction in the Kondo region, whose DC transport and shot noise have been measured recently [29].

We acknowledge funding by the ANR through Grants No. Project-ANR-16-CE30-0019 and No. Project-ANR-17-PIRE0001.
[1] I. K. Yanson, V. M. Svistunov, and I. M. Dmitrenko, Zh. Eksp. Teor. Fiz. 48, 976 (1965) [Sov. Phys. JETP 21, 650 (1965)].

[2] M. C. Cassidy, A. Bruno, S. Rubbert, M. Irfan, J. Kammhuber, R. N. Schouten, A. R. Akhmerov, and L. P. Kouwenhoven, Science 355, 939 (2017).

[3] A. Y. Kitaev, Phys. Usp. 44, 131 (2001).

[4] H. J. Kwon, K. Sengupta, and V. M. Yakovenko, Eur. Phys. J. B 37, 349 (2004).

[5] R. S. Deacon, J. Wiedenmann, E. Bocquillon, F. Domínguez, T. M. Klapwijk, P. Leubner, C. Brüne, E. M. Hankiewicz, S. Tarucha, K. Ishibashi, H. Buhmann, and L. W. Molenkamp, Phys. Rev. X 7, 021011 (2017).

[6] D. Laroche, D. Bouman, D. J. van Woerkom, A. Proutski, C. Murthy, D. I. Pikulin, C. Nayak, R. J. J. van Gulik, J. Nygård, P. Krogstrup, L. P. Kouwenhoven, and A. Geresdi, Nat. Commun. 10, 245 (2019).

[7] L. Fu and C. L. Kane, Phys. Rev. B 79, 161408(R) (2009).

[8] D. M. Badiane, M. Houzet, and J. S. Meyer, Phys. Rev. Lett. 107, 177002 (2011).

[9] D. I. Pikulin and Y. V. Nazarov, Phys. Rev. B 86, 140504(R) (2012).

[10] P. San-Jose, E. Prada, and R. Aguado, Phys. Rev. Lett. 108, 257001 (2012).

[11] J. D. Sau, E. Berg, and B. I. Halperin, arXiv:1206.4596.

[12] A fractional Josephson radiation without topological superconductivity has also been observed in a Cooper-pair transistor [30]. It was attributed to nonadiabatic Cooper-pair transfer.

[13] A. Martín-Rodero and A. Levy Yeyati, Adv. Phys. 60, 899 (2011).

[14] R. Žitko, Proc. SPIE 10732, 107321N (2018).

[15] V. Meden, J. Phys.: Condens. Matter 31, 163001 (2019).

[16] L. I. Glazman and K. A. Matveev, Pis'ma Zh. Eksp. Teor. Fiz. 49, 570 (1989) [JETP Lett. 49, 659 (1989)].
[17] E. Vecino, A. Martín-Rodero, and A. Levy Yeyati, Phys. Rev. B 68, 035105 (2003).

[18] A. Zazunov, S. Plugge, and R. Egger, Phys. Rev. Lett. 121, 207701 (2018).

[19] T. Matsuura, Prog. Theor. Phys. 57, 1823 (1977).

[20] C. W. J. Beenakker and H. van Houten, in Single-Electron Tunneling and Mesoscopic Devices, edited by $\mathrm{H}$. Koch and $\mathrm{H}$. Lübbig (Springer, Berlin, 1992), pp. 175-179.

[21] D. Averin and A. Bardas, Phys. Rev. Lett. 75, 1831 (1995).

[22] A. Levy Yeyati, A. Martín-Rodero, and E. Vecino, Phys. Rev. Lett. 91, 266802 (2003).

[23] M. Houzet, J. S. Meyer, D. M. Badiane, and L. I. Glazman, Phys. Rev. Lett. 111, 046401 (2013).

[24] M. Zgirski, L. Bretheau, Q. Le Masne, H. Pothier, D. Esteve, and C. Urbina, Phys. Rev. Lett. 106, 257003 (2011).

[25] Note that the states $(0,0)$ and $(1,1)$ are degenerate. They carry zero current (see Eq. (S2) of the SM [27]) and have the same incoming and outgoing probabilities [see Fig. 2(c)]. Hence, an alternative model not distinguishing these states could be formulated. However, it would be unsuited for the comparison with topological junctions (see Sec. 2 of the SM [27]).

[26] D. Averin and H. T. Imam, Phys. Rev. Lett. 76, 3814 (1996).

[27] See Supplemental Material at http://link.aps.org/supplemental/ 10.1103/PhysRevResearch.2.033158 for the derivation of Eqs. (14) and (15) and an alternative description of the junction at $p_{\pi}=1$, which clarifies the relation between our model and topological junctions.

[28] Y. Naveh and D. V. Averin, Phys. Rev. Lett. 82, 4090 (1999).

[29] T. Hata, R. Delagrange, T. Arakawa, S. Lee, R. Deblock, H. Bouchiat, K. Kobayashi, and M. Ferrier, Phys. Rev. Lett. 121, 247703 (2018).

[30] P. M. Billangeon, F. Pierre, H. Bouchiat, and R. Deblock, Phys. Rev. Lett. 98, 216802 (2007). 